\title{
Current Mechanisms in n-SiC/p-Si Heterojunctions
}

\author{
Philip Tanner, Sima Dimitrijev, H. Barry Harrison \\ Queensland Microtechnology Facility \\ Griffith University \\ Brisbane, Australia \\ $\underline{\text { P.Tanner@griffith.edu.au }}$
}

\begin{abstract}
Current-voltage characteristics of $3 \mathrm{C}-\mathrm{SiC} / \mathrm{Si}$ heterojunction diodes are presented with reverse breakdown voltage exceeding $200 \mathrm{~V}$, leakage current of $1.3 \mathrm{mAcm}^{-2}$ at $200 \mathrm{~V}$, and $a+/-1 V$ rectification ratio of 200,000 . The reverse leakage current was observed to have both temperature and field dependence and hence a model is presented to explain this observation based on a trap assisted tunneling-thermal emission mechanism located at the $\mathrm{SiC} / \mathrm{Si}$ interface. With further improvements in the $\mathrm{SiC}$ crystal quality at the silicon interface, trap concentrations and thus reverse bias leakage currents will be reduced.
\end{abstract}

Keywords-3C-SiC;heterojunction diode;current mechanism

\section{INTRODUCTION}

The $\mathrm{SiC} / \mathrm{Si}$ heterojunction has been investigated as a promising candidate in many semiconductor device applications, including high-voltage and high-frequency diodes [1],[2] heterojunction bipolar transistors [3],[4] and photovoltaic cells [5]. Due to the wide bandgap, high thermal conductivity, and chemical stability of $\mathrm{SiC}$, it is an excellent semiconductor to be combined with silicon in a heterojunction diode device. Yet, of the many different SiC polytypes that exist, only $3 \mathrm{C}-\mathrm{SiC}$ has been successfully grown on silicon substrates at temperatures below the melting point of silicon [6],[7]. Many different growth techniques and chemistries have been investigated in recent years, resulting in the reduction of crystal defects that occur due to the lattice and thermal mismatch between the epi-layer and the substrate. While thicker layers exhibit good crystal quality away from the silicon interface, this interface region remains a major source of structural and electronic defects. The valence band offset between $\mathrm{SiC}$ and silicon (1.7eV, [8]) forms a large barrier to hole injection and in the ideal case, the reverse saturation current should be extremely low, yet due to the high density of defects at the interface the diode characteristics are far from ideal.

In homojunctions and heterojunctions the established mechanisms of trap-assisted generation in the depletion region and band-to-band tunneling at high bias are well established [9]. Some studies find space charge region generation in $\mathrm{SiC} / \mathrm{Si}$ structures as evidenced by the $\mathrm{I} \mathrm{V}^{1 / 2}$ relationship [4], while others propose a mechanism of excess-tunneling to explain current-voltage characteristics of $\mathrm{n}-3 \mathrm{C}-\mathrm{SiC} / \mathrm{Si}$ heterojunctions [10]. In this paper, we focus on the current mechanism responsible for the reverse bias leakage and propose a new mechanism governed by trap assisted tunneling and thermal emission that gives rise to both temperature and field dependence of the reverse current.

\section{DEVICE FABRICATION AND TESTING}

3C-SiC/Si samples were supplied by QsSemi and fabricated at the Queensland Microtechnology Facility. Single crystal ntype $3 \mathrm{C}-\mathrm{SiC}$ was grown to a thickness of $500 \mathrm{~nm}$ on $25 \Omega-\mathrm{cm}$, p-type $<100>$ silicon in a hot wall CVD furnace at $1100^{\circ} \mathrm{C}$ with n-type doping concentration in the range $1-5 \times 10^{16} \mathrm{~cm}^{-3}$. The SiC surface was cleaned with acetone, isopropanol, and then $1 \% \mathrm{HF}$ before depositing aluminium and patterning into $500 \mu \mathrm{m}$ by $230 \mu \mathrm{m}$ ohmic contacts with a surrounding guard ring to reduce surface leakage currents. The back silicon surface was etched in HF and aluminium deposited as a back ohmic contact. Contact series resistance was not optimized in this study. Current-voltage measurements were carried out in a light-tight electrically shielded enclosure on a temperature controlled hotchuck.

\section{RESULTS AND DISCUSSION}

Fig. 1 shows the room temperature current-voltage characteristics of a heterojunction diode. The forward bias current density obeys the relationship $\mathrm{J}=\mathrm{J}_{0}$. $\exp (\mathrm{qV} / \mathrm{nkT})$ where the ideality factor $n=1.2$. When diffusion current dominates, $\mathrm{n}=1$ and when recombination dominates, $\mathrm{n}=2$ [9]. Thus, an ideality factor of 1.2 indicates that the forward bias current is dominated by diffusion but also contains a component of recombination, most likely to occur at the $\mathrm{SiC} / \mathrm{Si}$ interface. The reverse saturation current density $\mathrm{J}_{0}=0.1 \mu \mathrm{A} / \mathrm{cm}^{2}$ at room temperature and increasing temperatures reveal an activation energy for $\mathrm{J}_{0}$ of $0.91 \mathrm{eV}$.

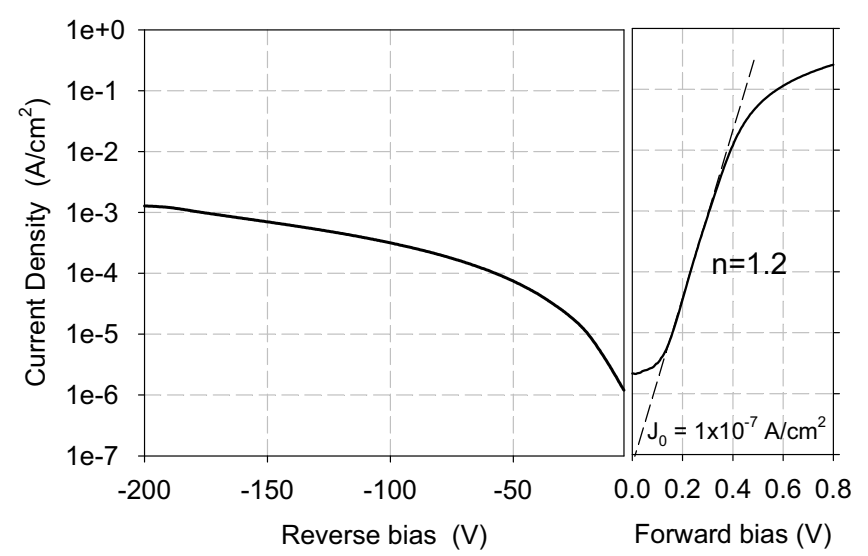

Figure 1. Current-voltage characteristics of $n-3 \mathrm{C}-\mathrm{SiC} / \mathrm{p}-\mathrm{Si}$ heterojunction in forward and reverse bias. In forward bias, the ideality factor is 1.2 and $\mathrm{J}_{0}$ is $0.1 \mu \mathrm{A} / \mathrm{cm}^{2}$. Note the different voltage scales for reverse and forward bias. 
The diode shows excellent reverse blocking characteristics, with no apparent breakdown to $200 \mathrm{~V}$, where the current density is $1.3 \mathrm{~mA} / \mathrm{cm}^{2}$ and the rectification ratio at $+/-1 \mathrm{~V}$ is 200,000.

Two important properties of a heterojunction diode are the reverse leakage current and breakdown voltage. Modern silicon wafers are extremely pure and hence the contribution of bandgap traps to the generation of minority carriers in the depletion region is expected to be very low compared with that of defects at the $\mathrm{SiC} / \mathrm{Si}$ junction. The reverse bias current was seen not to be a linear function of $\left(\mathrm{V}_{\mathrm{bi}}+\mathrm{V}\right)^{1 / 2}$, confirming that space charge region generation is not the dominant mechanism. Fig. 2 shows the temperature dependence of the reverse bias current, at a number of different voltages. Since the doping of the $\mathrm{SiC}$ is orders of magnitude higher than the silicon, most of the electric field change occurs in the silicon near the interface. It is evident that the activation energy, calculated from the slope of the reverse current versus $1 / \mathrm{kT}$ plot is not constant as the reverse bias is increased. Increasing the reverse bias from $5 \mathrm{~V}$ to $40 \mathrm{~V}$ results in a decrease in activation energy from $0.54 \mathrm{eV}$ to $0.39 \mathrm{eV}$ respectively. This combination of temperature and field dependence suggests that current generation is occurring via a multistep mechanism at the $\mathrm{SiC} / \mathrm{Si}$ interface rather than simply trap assisted thermal generation in the silicon depletion region or at the $\mathrm{SiC} / \mathrm{Si}$ interface.

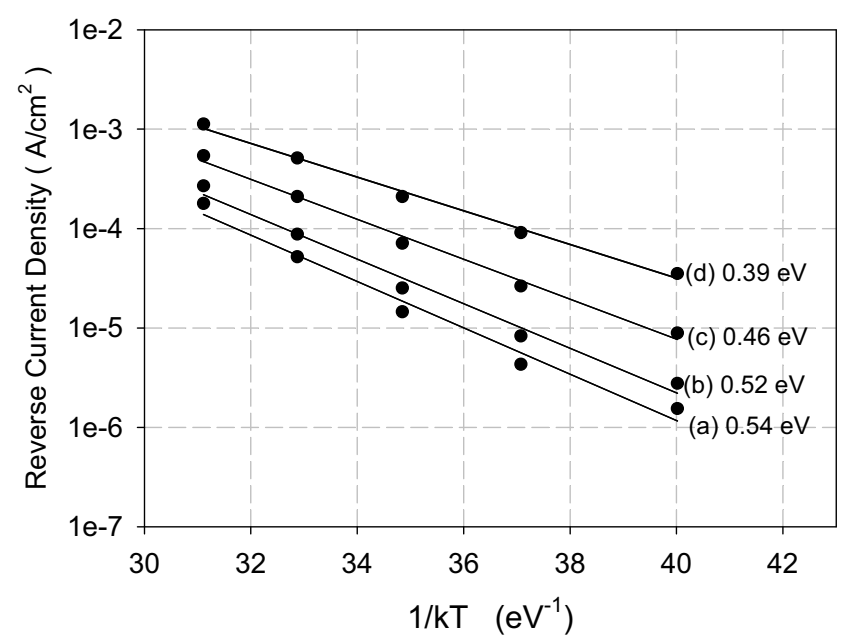

Figure 2. Arrhenius plots of the reverse current density measured at different bias voltages. (a) $5 \mathrm{~V}$, (b) $10 \mathrm{~V}$, (c) $20 \mathrm{~V}$, (d) $40 \mathrm{~V}$. The activation energy is shown for each voltage.

Fig. 3 shows the band alignment of the ideal heterojunction under zero bias conditions with values of $3 \mathrm{C}$ SiC bandgap and electron affinity obtained from Ref. [8]. To explain our experimental results in reverse bias, we propose a current mechanism that consists of an electron tunneling from the valence band of silicon to a trap at the $\mathrm{SiC} / \mathrm{Si}$ interface followed by thermal emission into the conduction band of the SiC. As shown in Fig. 4, when the reverse bias increases from lower values (black lines) to higher values (grey lines), the tunneling process occurs at energies further from the silicon valence band edge, thus reducing the energy required for thermal emission.

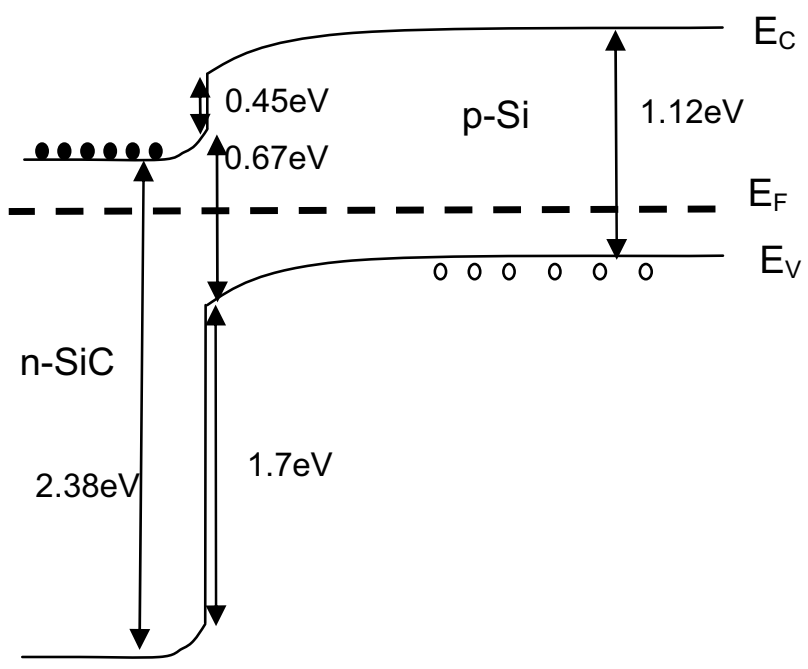

Figure 3. Band alignment diagram of the $\mathrm{n}-3 \mathrm{C}-\mathrm{SiC} / \mathrm{p}-\mathrm{Si}$ heterojunction at zero bias.

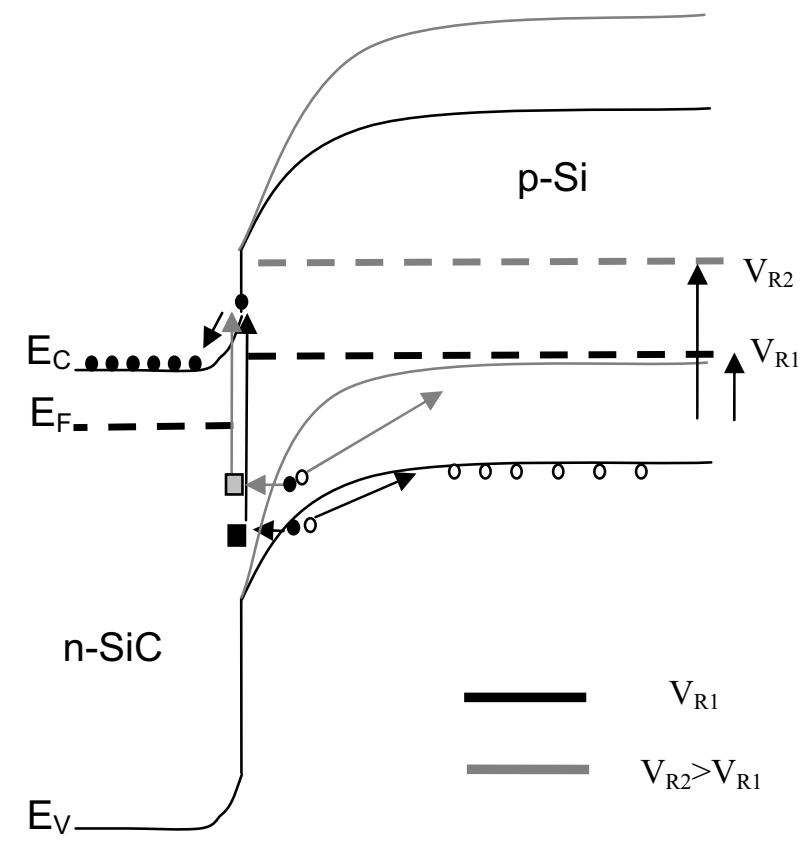

Figure 4. Band diagram showing how different values of reverse bias produce a two-step tunneling/thermal emission process that is favored by traps of different energies. 
Thus the current mechanism in reverse bias can be expressed by the following well established relationships [9]. The thermal emission time constant is governed by (1):

$$
\tau_{e}=\tau_{e 0} \cdot \exp \left[\frac{\left(E_{c, S i C}-E_{t}\right)}{k T}\right]
$$

where $\tau_{\mathrm{e} 0}$ is a constant, $\mathrm{E}_{\mathrm{c}, \mathrm{SiC}}$ is the $\mathrm{SiC}$ conduction band energy, $\mathrm{E}_{\mathrm{t}}$ the trap energy, $\mathrm{k}$ is Boltzman's constant, and $\mathrm{T}$ is temperature.

The tunneling process time constant is governed by (2):

$$
\tau_{t}=\tau_{t 0} \cdot \exp \left(\frac{\Phi_{B}^{3 / 2}}{E_{S i}}\right)
$$

where $\tau_{\mathrm{t} 0}$ is a constant, and $\mathrm{E}_{\mathrm{si}}$ is the electric field strength in the silicon at the heterojunction and $\Phi_{\mathrm{B}}$ is the tunneling barrier height. Thus, the total time for this two step process is:

$$
\tau=\tau_{e}+\tau_{t}
$$

Whichever trap energy provides the minimum value of $\tau$ will be chiefly responsible for the reverse current density $\mathrm{J}_{\mathrm{r}}$, according to the following equation:

$$
J_{r}=\frac{q N_{t}}{\tau}
$$

where $\mathrm{N}_{\mathrm{t}}$ is the trap density at that energy.

\section{CONCLUSIONS}

We have demonstrated n-3C-SiC / p-Si heterojunctions with reverse bias breakdown voltages exceeding $200 \mathrm{~V}$, and $+/-1 \mathrm{~V}$ rectification ratio of 200,000 at room temperature. The forward bias current was found to be dominated by diffusion, with some proportion of recombination present at the $\mathrm{SiC} / \mathrm{Si}$ interface as indicated by a diode quality factor of 1.2. Temperature and field dependence of the reverse diode current is explained by a trap-assisted, tunneling/thermal emission mechanism at the $\mathrm{SiC} / \mathrm{Si}$ interface. Electrons tunnel from the valance band of the silicon to the most favorable trap level and are then thermally emitted to the conduction band of the SiC. As the electric field in the silicon increases with reverse bias, the tunneling process is assisted and the energy of thermal emission is reduced. Future reduction of crystal defects at this interface will result in further improvements in reverse bias leakage currents.

\section{ACKNOWLEDGMENT}

The authors would like to thank QsSemi for supplying the samples for this study.

\section{REFERENCES}

[1] P.H. Yi, J.P. Li, A.J. Steckl, "SiC/Si heterojunction diodes fabricated by self-selective and by blanket rapid thermal chemical vapour deposition", IEEE Trans. Electron Dev., Vol. 41, No. 3, Page 281, 1994.

[2] J. Perzolt, Ch. Foster, P. Weih, P.Masri, "Electrical characterization of $\mathrm{SiC} / \mathrm{Si}$ heterostructures with Ge-modified interfaces”, Appl. Surf. Sci., Vol. 184, Issues 1-4, Pages 79-83, 2001.

[3] M. Jugadesh Kumar and C.L. Reddy, "Realising wide bandgap P-SiCemitter lateral heterojunction bipolar transistors with low collector-emitter offset voltage and high current gain: a novel proposal using numerical simulation", IEE Proc. Circuits Devices Syst., Vol. 151, No. 5, Page 399, October 2004,

[4] M.I. Chaudhry, "Electrical transport properties of crystalline silicon carbide/silicon heterojunctions", IEEE Electron Dev. Let. , Vol. 12, No.12, Pg $670,1991$.

[5] Shunsuke Ogawa, Norimitsu Yoshida, Takashi Itoh ${ }^{1}$, and Shuichi Nonomura, "Heterojunction Amorphous Silicon Solar Cells with n-Type Microcrystalline Cubic Silicon Carbide as a Window Layer", Jpn. J. appl. Phys., Vol. 46, Page 518-522, 2007.

[6] H. Nagasawa and K. Yagi, "3C-SiC single crystal films grown on 6-inch Si substrates", Phys. Status Solidi B, Vol. 202, Page 335, 1997.

[7] Thierry Chassagne, Gabriel Ferro, Didier Chaussende, François Cauwet, Yves Monteil and Jean Bouix, "A comprehensive study of SiC growth processes in a VPE reactor”, Thin Solid Films, Vol. 402, pages 83-89, 2002.

[8] V.V. Afanas'ev, M. Bassler, G. Pensl, M.J. Schulz, "Band offsets and electronic structure of $\mathrm{SiC} / \mathrm{SiO} 2$ interfaces"; J. Appl. Phys. 79, Pg 3108, 1996.

[9] Sze, "Physics of Semiconductor Devices", 2 $2^{\text {nd }}$ Edition, Wiley Interscience, 1981.

[10] S. Zh. Karazhanov, I.G. Atabaev, T.M. Saliev, E.V. Kanaki, E. Dzhaksimov, "Excess tunneling currents in p-Si-n-3C-SiC heterostructures", Semiconductor Vol. 35, No. 1, Page 75-77, 2001. 